\title{
THE EUPHROSYNE FAMILY'S CONTRIBUTION TO THE LOW ALBEDO NEAR-EARTH ASTEROIDS
}

\author{
Joseph R. Masiero $^{1}$, V. Carruba ${ }^{2}$, A. Mainzer ${ }^{1}$, J. M. Bauer ${ }^{1}$, and C. Nugent ${ }^{3}$ \\ ${ }^{1}$ Jet Propulsion Laboratory/Caltech, 4800 Oak Grove Dr., MS 183-601, Pasadena, CA 91109, USA; \\ Joseph.Masiero@jpl.nasa.gov, amainzer@jpl.nasa.gov, bauer@scn.jpl.nasa.gov \\ 2 Departamento de Matemática, Universidade Estadual Paulista, Av. Dr. Ariberto Pereira da Cunha 333. \\ CEP 12516-410, Guaratinguetá, SP, Brazil; vcarruba@feg.unesp.br \\ ${ }^{3}$ Infrared Processing and Analysis Center, Caltech, Mail Code 100-22, 770 South Wilson Avenue, \\ Pasadena, CA 91125, USA; cnugent@ipac.caltech.edu \\ Received 2015 May 22; accepted 2015 July 17; published 2015 August 21
}

\begin{abstract}
The Euphrosyne asteroid family is uniquely situated at high inclination in the outer Main Belt, bisected by the $\nu_{6}$ secular resonance. This large, low albedo family may thus be an important contributor to specific subpopulations of the near-Earth objects. We present simulations of the orbital evolution of Euphrosyne family members from the time of breakup to the present day, focusing on those members that move into near-Earth orbits. We find that family members typically evolve into a specific region of orbital element-space, with semimajor axes near $\sim 3 \mathrm{AU}$, high inclinations, very large eccentricities, and Tisserand parameters similar to Jupiter family comets. Filtering all known Near-Earth objects (NEOs) with our derived orbital element limits, we find that the population of candidate objects is significantly lower in albedo than the overall NEO population, although many of our candidates are also darker than the Euphrosyne family, and may have properties more similar to comet nuclei. Followup characterization of these candidates will enable us to compare them to known family properties, and confirm which ones originated with the breakup of (31) Euphrosyne.
\end{abstract}

Key words: minor planets, asteroids: general

\section{INTRODUCTION}

The Euphrosyne asteroid family occupies a unique place in orbital element space among families, located in the outer Main Belt at very high inclination, as shown in Figure 1. It is also the only asteroid family that is bisected by the $\nu_{6}$ secular orbital resonance. The inner-most portion of this resonance (semimajor axis $<2.5 \mathrm{AU}$ ) has been shown to be a primary mechanism for moving asteroids onto near-Earth orbits with relatively long lifetimes (Bottke et al. 2002). Conversely, Bottke et al. (2002) found that the Jupiter mean motion resonances tended to quickly move objects onto Jupiter-crossing orbits that lead to an impact or ejection from the Solar system.

The largest remnant of this family, (31) Euphrosyne, is one of the largest asteroids in the Main Belt, with a diameter of $D=260 \pm 12 \mathrm{~km}$, an optical albedo of $p_{V}=0.05 \pm 0.01$ (Masiero et al. 2014), a bulk density of $\rho=1.2 \pm 0.6 \mathrm{~g} \mathrm{~cm}^{-3}$ (Carry 2012), and a taxonomic classification of $\mathrm{Cb}$ (Bus \& Binzel 2002). The Euphrosyne family is one of the most numerous families, with approximately 1000 associated members (Nesvorný 2012; Milani et al. 2014). The family has an average albedo of $p_{V}=0.056 \pm 0.016$, very close to that of the largest remnant, and an anomalously steep present day cumulative size frequency distribution (SFD) of $N(>D) \propto D^{-4.4}$ (Masiero et al. 2013). As one of the few large, low-albedo families near the $\nu_{6}$ resonance, Euphrosyne may be one of the important contributors to the low albedo component of the Near-Earth object (NEO) population (cf. Mainzer et al. 2011a).

In this work, we investigate the potential contribution of the Euphrosyne family to the NEO population over the predicted $\sim 700 \mathrm{Myr}$ age of the family (Carruba et al. 2014). We quantify the most likely region of orbital-element phase space that these family escapees would be found in, and search the list of known NEOs for candidate objects related to the family. We also produce a shortlist of followup candidates that have a high likelihood of being compositionally related to the Euphrosyne family.

\section{SIMULATIONS}

To test the contribution of the Euphrosyne family to the near-Earth population, we performed simulations of the orbital evolution of the small asteroids created by the catastrophic impact event that formed the observed family. We use the SWIFT-RMVSY symplectic numerical integrator (Holman \& Wisdom 1993; Levison \& Duncan 1994; Brož 2006) to follow the evolution of these test particles under both gravity and the Yarkovsky effect (Bottke et al. 2006). We performed seven independent simulations each consisting of 4000 massless test particles, for a total of 28,000 simulated family members. For massive bodies in each simulation we used the Sun, Mercury, Venus, the Earth-Moon barycenter, Mars, Jupiter, and Saturn. Test simulations carried out using all planets as massive bodies show identical results to the restricted case; thus, Uranus and Neptune were excluded to reduce run time of the simulations. Positions for the massive bodies were initialized to their computed location on MJD $=57,000$ from the JPL DE405 planetary ephemerides file. ${ }^{4}$

Family members were initialized at the position of (31) Euphrosyne on MJD $=57000$ and given a randomized velocity relative to the parent body. The magnitude of the velocity was scaled inversely proportionally to the diameter of the test particle (Vokrouhlický et al. 2006), with a characteristic velocity of $130 \mathrm{~m} \mathrm{~s}^{-1}$ for an object with a diameter $D=5 \mathrm{~km}$, following the best fit breakup velocity found by Carruba et al. (2014). A secondary phase space minimum is present for a significantly lower characteristic velocity of $\sim 20 \mathrm{~m} \mathrm{~s}^{-1}$ (Carruba et al. 2015), so we also simulated a set of test cases

\footnotetext{
4 http://ssd.jpl.nasa.gov/?planet_eph_export
} 


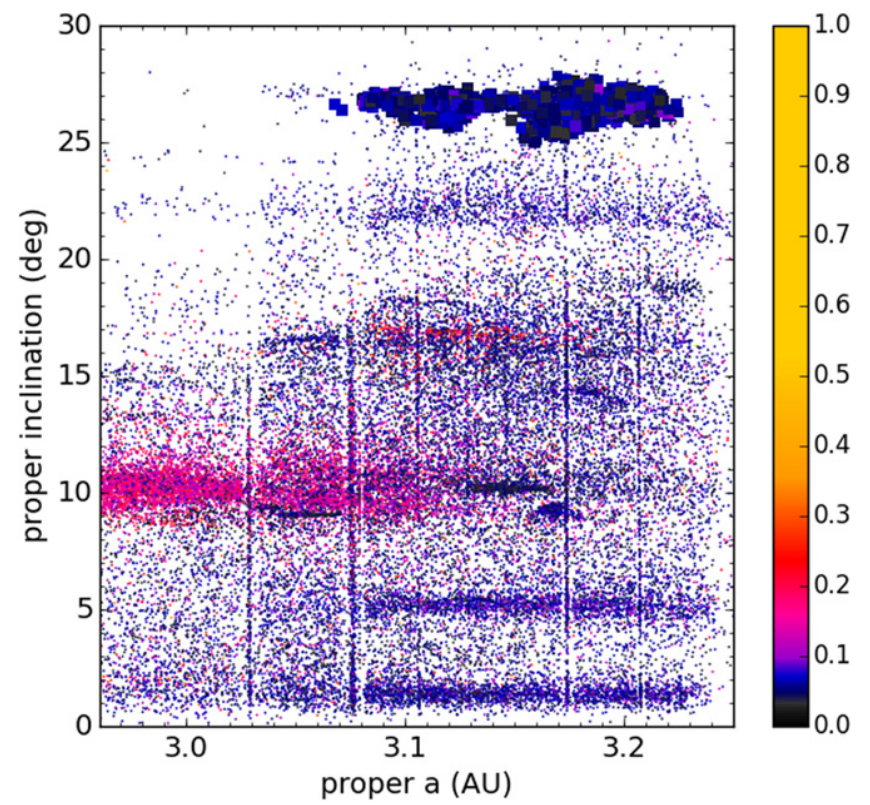

Figure 1. Proper orbital inclination vs. proper semimajor axis for all asteroids in the outer Main Belt (dots) and Euphrosyne family members (large squares). Colors indicate visible geometric albedo following the colorbar. Vertical features are a result of weak resonances and a feature common to synthetic proper orbital element catalogs. Horizontal features are asteroid families. Orbital elements, albedos, and family identifications taken from Masiero et al. (2013).

with a characteristic velocity of $20 \mathrm{~m} \mathrm{~s}^{-1}$ to look for potential differences in the final result that could result from a variation in this initial condition.

The physical properties assumed for the test particles, which play an important role in the strength of the Yarkovsky effect, were drawn from the overall characteristics of the family. The geometric albedo of each object was assumed to be $p_{V}=0.056$, matching the mean for the family given by Masiero et al. (2013). Bulk and surface densities were assumed to be $1200 \mathrm{~kg} \mathrm{~m}^{-3}$, based on the measured values for the largest remnant as well as for asteroids of similar spectral taxonomic type (Carry 2012). As full thermophysical models of (31) Euphrosyne or Euphrosyne family members are not available, we assume nominal thermophysical parameter values for the simulated particles based on previous research (Vokrouhlický et al. 2006): thermal capacity $C_{p}=680 \mathrm{~J} \mathrm{~kg}^{-1} \mathrm{~K}^{-1}$, emissivity $\epsilon=0.9$, and thermal conductivity $K=0.01 \mathrm{~W} \mathrm{~m}^{-1} \mathrm{~K}^{-1}$. The evolution of our test particles is dominated by secular resonances and other gravitational effects in this case, and Yarkovsky orbital evolution is not required to inject particles into these resonances, thus the uncertainty in these thermal parameter values does not dominate the simulation results.

As discussed by Carruba et al. (2014), the slope of the family size frequency distribution changes as the family evolves. Thus, sizes for simulated particles were generated to fulfill a cumulative size frequency distribution slope of $\alpha=-3.6$, based on the best-fitting initial value from Carruba et al. (2014) over the range from 1 to $20 \mathrm{~km}$. Test particles were given random initial spin states, which evolve under YORP and collisional re-randomization as described in Brož (2006). Bottke et al. (2015) introduce a new method for simulating YORP evolution they refer to as "stochastic" YORP, however given the proximity of the family to the $\nu_{6}$ resonance, this new effect is not expected to significantly alter the results as

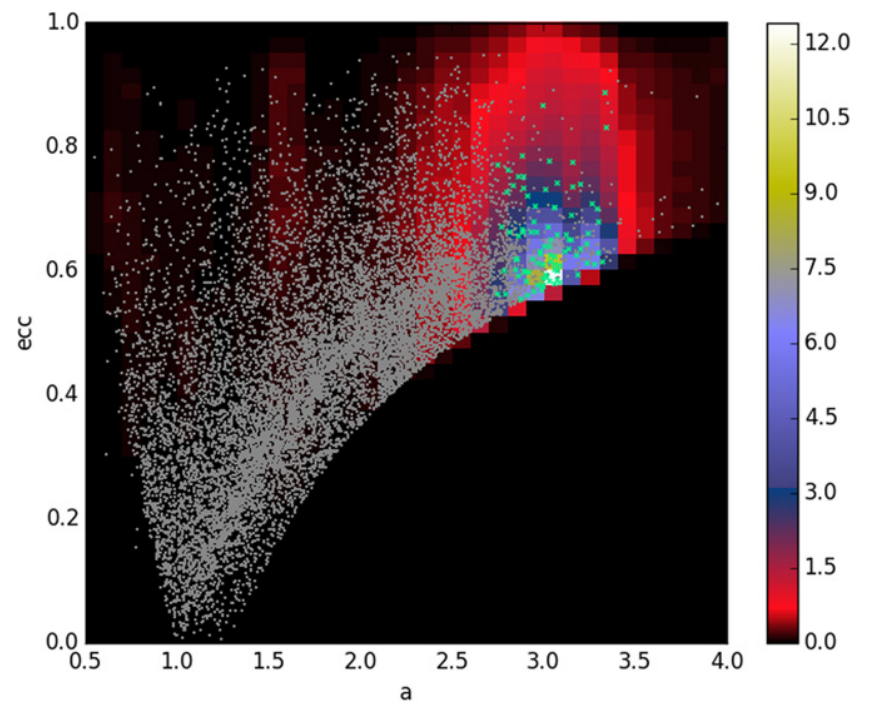

Figure 2. Density map of orbital elements of all simulated Euphrosyne family members entering the near-Earth population. Bins of semimajor axis (a) and eccentricity (ecc) are colored according to the probability density shown in the colorbar. Overplotted as points are all known NEOs in gray, with those inside our selection criteria in green.

compared to the older YORP simulation technique employed here.

Simulations were run forward for $700 \mathrm{Myr}$, using 3 day time steps to ensure that minimal errors would be induced in the orbit of Mercury or any test particle with a small perihelion distance. We recorded the orbit of each particle every $10 \mathrm{kyr}$ over the entire simulation, and selected all objects with perihelia $q<1.3 \mathrm{AU}$, which would be classified as NEOs. We then tracked the orbital elements of each simulated NEO to look for characteristic values that would allow us to search the real NEO population for potential Euphrosyne family members.

\section{RESULTS}

Our simulations of family member evolution show that the primary path for entry into the NEO population is a slow pumping of orbital eccentricity by resonances, while semimajor axis and inclination remain similar to the initial family values. Simulations using the lower initial breakup velocity show nearly identical evolution in all respects, so we only discuss notable differences between the two initial condition cases. Figures 2-5 show the probability densities of orbital parameters for Euphrosyne family members that become NEOs, with probabilities shown as the coloring of the bin. At each time step each object present in the NEO population was recorded in the appropriate bin based on its orbit to form a probability density. Bin values, as displayed in the figures, were normalized such that the total probability sums to 1 . The highest density of particles is present at a semimajor axis range slightly smaller than the location of the family $(a \sim 2.9-3.1$ AU versus $3.08<a<3.22 \mathrm{AU}$ for the family) and at slightly lower inclinations (inc $\sim 18^{\circ}-24^{\circ}$ versus $24^{\circ}<$ inc $<27^{\circ}$ for the family), but at significantly larger eccentricities.

As the core of the Euphrosyne family intersects the $\nu_{6}$ resonance, a large fraction of our test particles $(\sim 80 \%)$ are evacuated from the family region and reach near-Earth space. Thus, the Euphrosyne family observed today is likely significantly less numerous in the tested size ranges than the initial family formed at impact. Unusually, smaller objects are 


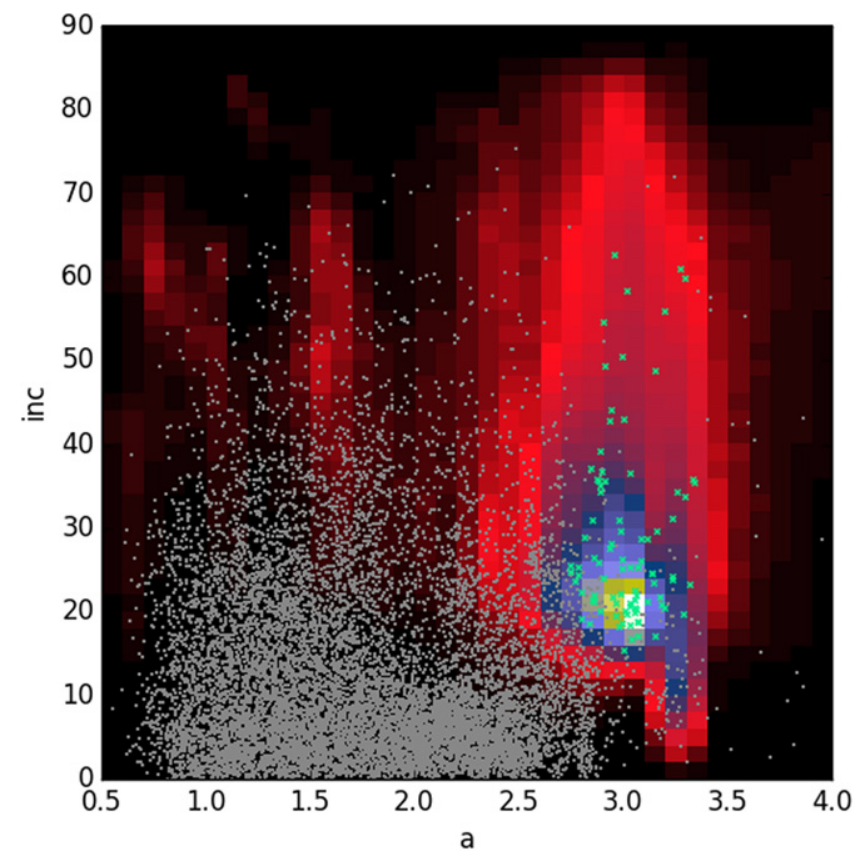

Figure 3. Same as Figure 2 but showing orbital inclination (inc) vs. semimajor axis $(a)$.

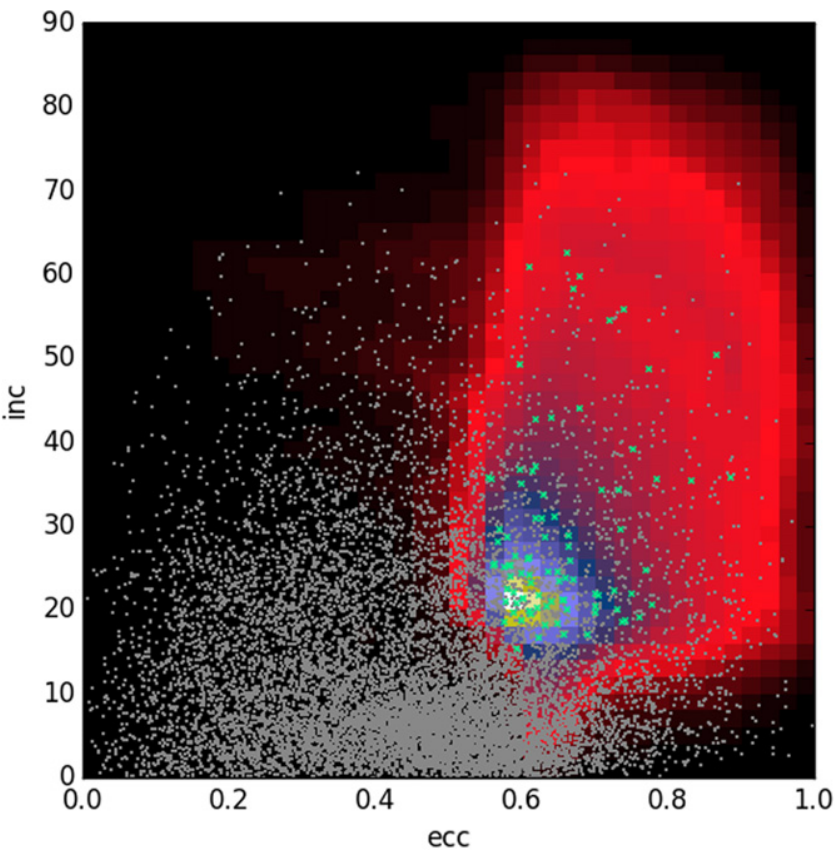

Figure 4. Same as Figure 2 but showing orbital inclination (inc) vs. eccentricity (ecc).

less likely to be removed from the family, as the larger initial velocity imparted during formation will move them further from the region of the $\nu_{6}$.

Once in the NEO population, the Kozai mechanism can result in exchanges between orbital eccentricity and orbital inclination (Kozai 1962), creating the fan-shaped pattern in Figure 4, though the majority of particles remain near $a \sim 3$ AU, $e \sim 0.6$, and $i \sim 20^{\circ}$. Nearly all particles in our simulation entering the NEO population have a Tisserand parameter $T_{\mathrm{J}}<3$. This value for the Tisserand parameter can potentially indicate a history with the potential for gravitational interaction

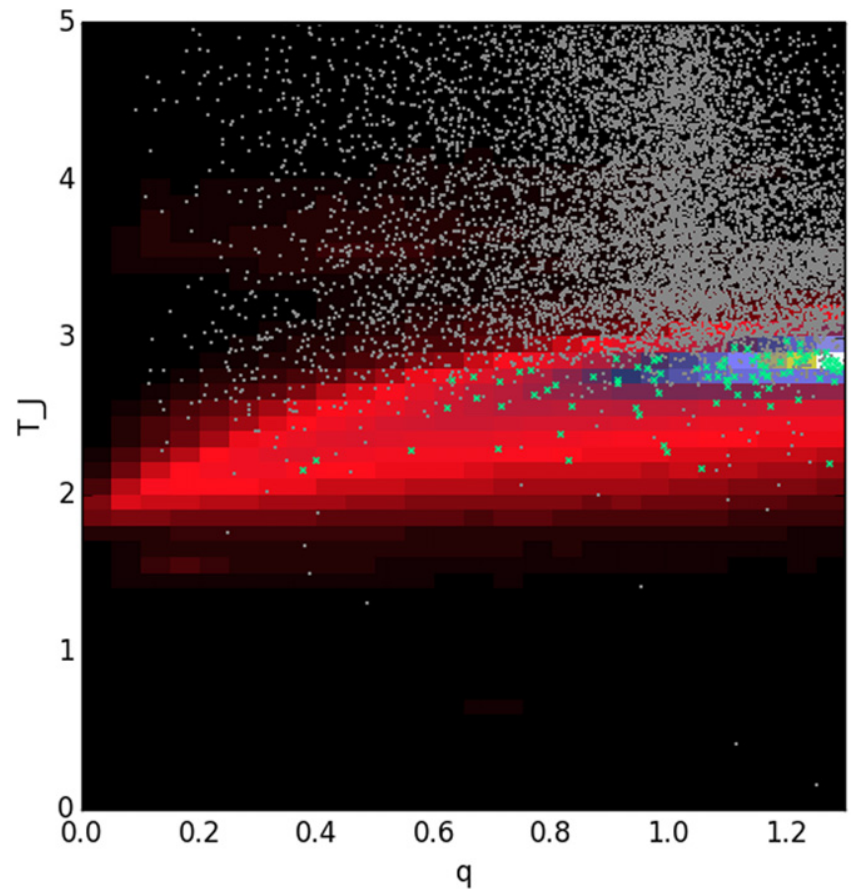

Figure 5. Same as Figure 2 but showing Tisserand parameter $\left(T_{\mathrm{J}}\right)$ vs. perihelion distance $(q)$.

with Jupiter and is often used to grade cometary orbits (Levison 1996).

These results, however, show the probability density over the entire timescale of the simulation. In order to compare our results to the present-day NEO population, we need to consider the evolution of orbital element properties of the test particles in the NEO region over time. Particles entering this region of phase space near the end of the simulation will be the best analogs for real objects that may be currently present in the NEO region.

In Figure 6 we show the orbital element evolution of all family members becoming NEOs in our simulations. Each point represents the instantaneous orbital elements of an object every $10 \mathrm{kyr}$. We also show a running mean of all objects for each element. The orbital elements of these NEOs do not change dramatically over the timescale of our simulations, but instead show a population that is in a rough steady-state, though with falling number density. Most of the particles leave the NEO population when their eccentricity approaches ecc $=1.0$ and they impact the Sun or are ejected from the Solar system. We note that a handful of our test objects evolved into Earth-like orbits, which were significantly more stable than the majority of our population. As such, these particles exert an overly large influence on the running means, and explain the occasional large deviations of the mean from the nominal behavior of the rest of the population. Other than these outlier objects, the majority of the population is found in narrow orbital ranges, particularly for semimajor axis and Tisserand parameter, while eccentricity and inclination both show fairly strong lower boundaries. As the mean parameters do not change over the course of the simulation, we can confidently use the overall probability density maps to search for objects in the present day NEO population.

Euphrosyne family members that are injected into the NEO population typically have both high eccentricities and high 

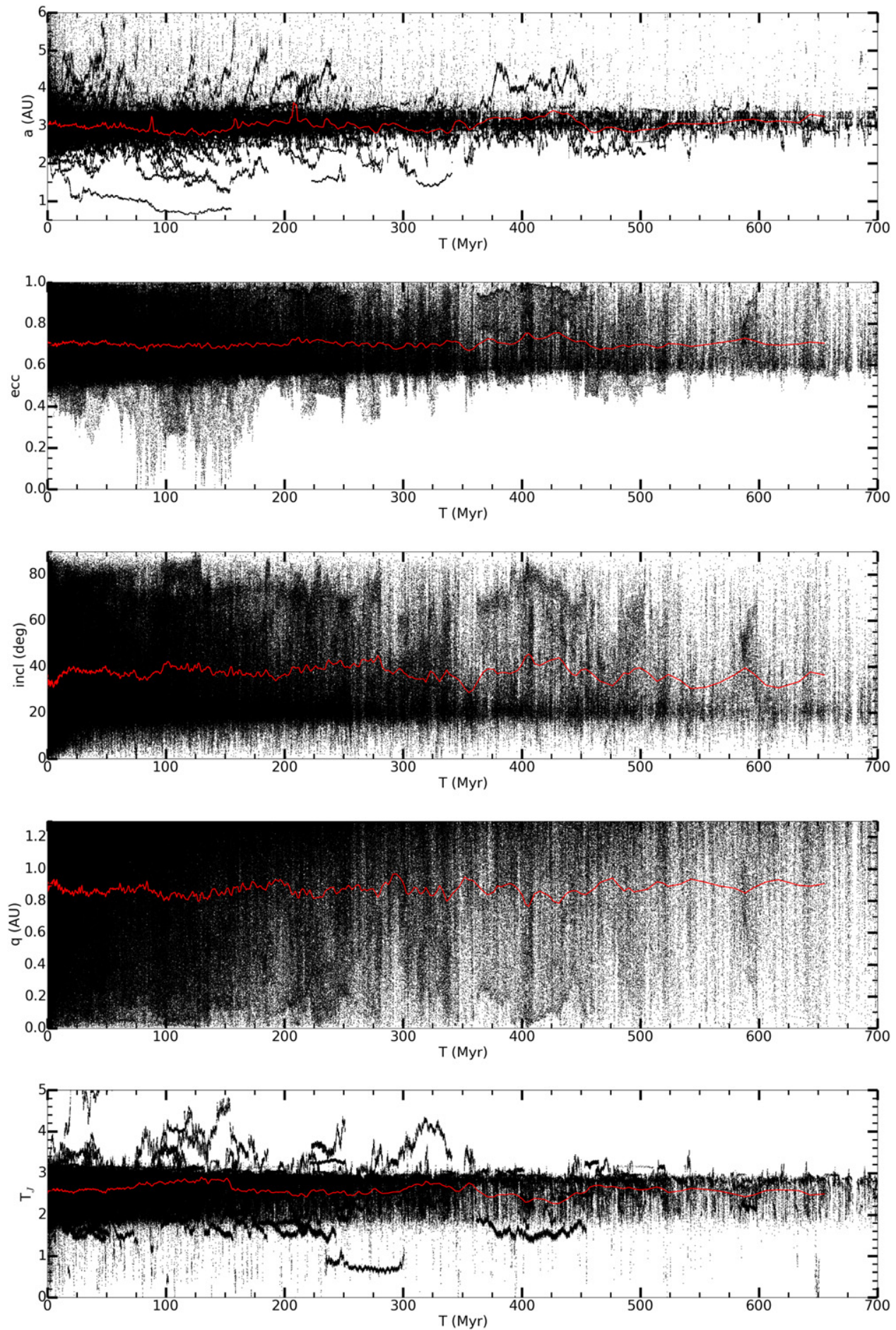

Figure 6. Orbital elements for all family members in the NEO population, recorded every $10 \mathrm{kyr}$ (points), as a function of time from the start of the simulation. The red line shows a running box mean of each element.

inclinations. As such, these objects will spend a majority of their orbit at a significant distance from the plane of the ecliptic. We investigated the characteristic vertical distances from the plane of the ecliptic of our test particles at perihelion $\left(\left|z_{\text {peri }}\right|\right)$ as a possible means of distinguishing objects related to the Euphrosyne family from background NEOs. Figure 7 shows the average $\left|z_{\text {peri }}\right|$ for all simulated objects over seven different time bins, compared to the distribution for all known NEOs. The $z_{\text {peri }}$ distribution for test particles does not change significantly over the timespan of the simulation, and shows a significantly larger value than what is observed for the known NEO population. We can thus use this parameter as an additional discriminant for identification of candidate family members.

We also investigate the lifetime of family members that migrate to the NEO population. A longer-lived population would be expected to make up a larger fraction of the phase space of interest than a population that rapidly transitions out of 


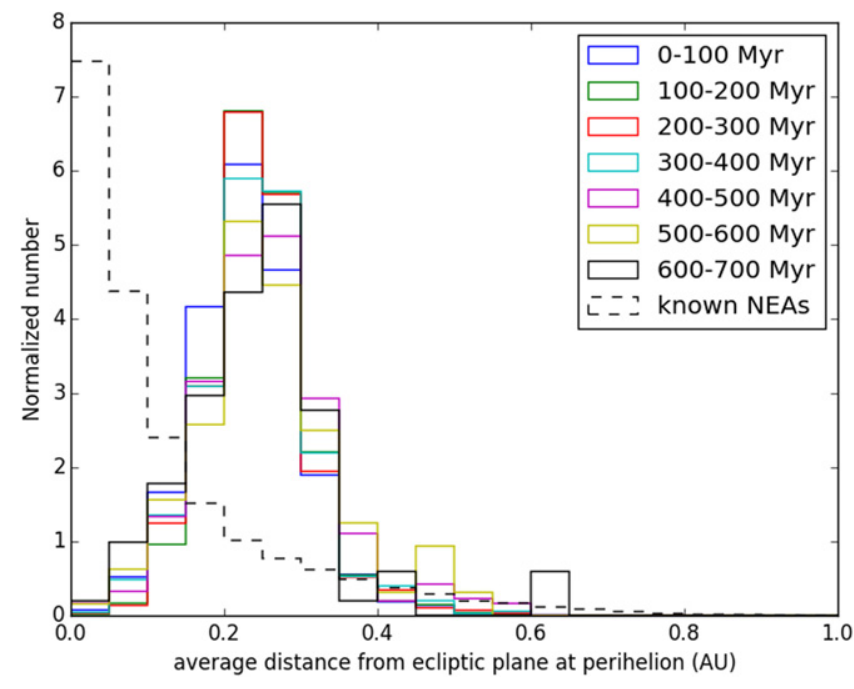

Figure 7. Distribution for simulated particles of the average distance from the ecliptic plane at the time of perihelion. The solid lines show the distribution for each 100 Myr time span of the simulation, while the dashed line shows the ecliptic plane distance for all currently known NEOs.

this region. Gladman et al. (2000) found that the median lifetime of NEOs was $\sim 10 \mathrm{Myr}$. However, the population investigated by those authors was based on all known objects at the time, which the authors point out was biased against the high inclination objects that dominate our sample. Thus, we would expect our objects to have potentially major differences from the population tested there.

We tabulate the lifetime of our simulated objects that enter the NEO population from their first entrance until they are removed (usually via ejection or Solar collision as eccentricity is increased to $e=1$ ). A subset of our population transitioned multiple times between near-Earth and Mars-crossing orbits before settling into the NEO population; however, this does not significantly impact our overall lifetime determination. The median lifetime and one-sigma range determined from our simulations was $875_{-440}^{+770} \mathrm{kyr}$, with only $2 \%$ of test particles surviving for more than 10 Myr. Figure 8 shows the distribution of particle lifetimes in our simulations. The most common endof-life for these objects is ejection from the Solar system or impact with the Sun when their eccentricity is increased near ecc $=1.0$. No difference was found in NEO lifetime between the two different initial velocity cases tested.

There was, however, one case of a significant difference between the two initial breakup velocities we simulated. In Figure 9 we show the cumulative number of objects transported from the family breakup into the NEO population over the course of the simulation. The faster initial breakup velocity resulted in a larger number of objects being injected onto nearEarth orbits on short timescales. However, the total population of objects transitioning into NEOs over the time since the breakup of the family ( $700 \mathrm{Myr})$ is not significantly different.

\section{DISCUSSION}

Our simulations have shown that the breakup of the Euphrosyne family resulted in a population of objects in near-Earth space with characteristic orbital elements that are different from the majority of NEOs. The span of initial breakup velocities behaves nearly identically in orbital element evolution, and results in comparable contribution to the present

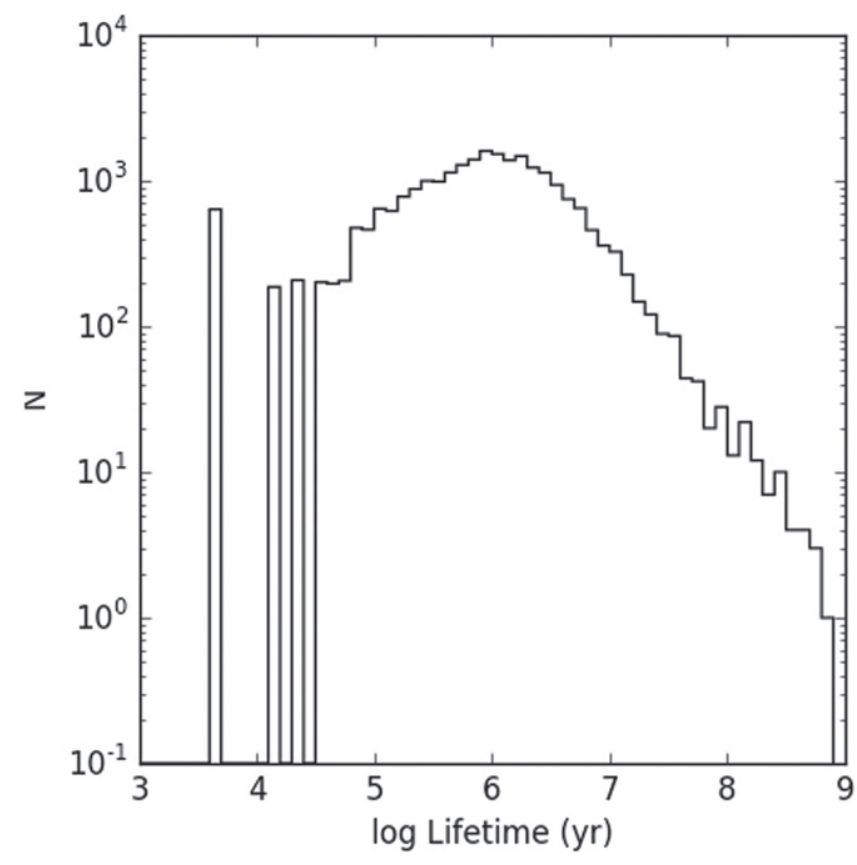

Figure 8. Distribution of lifetimes within the NEO population for evolving family members, totaling 24,000 particles.

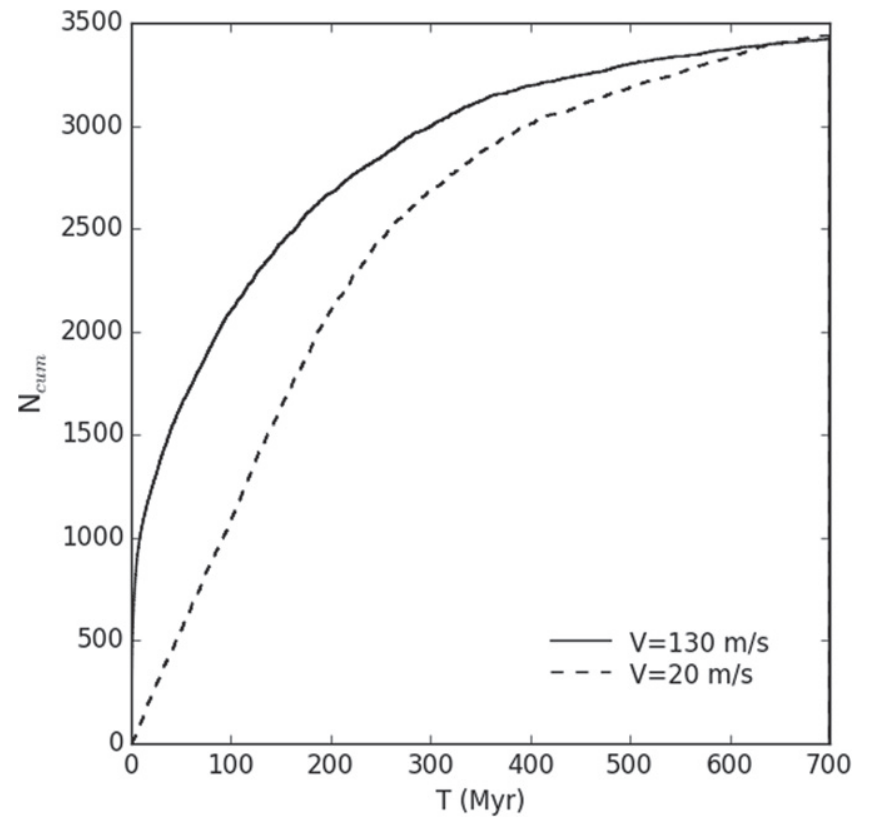

Figure 9. Cumulative number of test particles from the Euphrosyne family injected into the NEO population over the entire timescale of the simulation and estimated family age. The solid line shows simulations using a characteristic breakup velocity of $V=130 \mathrm{~m} \mathrm{~s}^{-1}$, while the dashed line shows the slower initial condition of $V=20 \mathrm{~m} \mathrm{~s}^{-1}$ that was also tested.

NEO population, though the historical rate is significantly different. We can use the characteristic orbital elements to search the known list of near-Earth objects for candidate family members.

We perform multi-dimensional cuts in orbital element space based on the highest-likelihood regions of the simulations to search for candidate objects. Our orbital element cuts to determine candidates were:

$$
\text { 1. } 2.75<a<3.35 \mathrm{AU}
$$


Table 1

Candidate Euphrosyne Family Members on NEO Orbits Having NEOWISE-measured Physical Properties

\begin{tabular}{|c|c|c|c|c|c|c|}
\hline $\begin{array}{c}\text { Name } \\
\text { MPC-packed Format }\end{array}$ & $\begin{array}{c}a \\
(\mathrm{AU})\end{array}$ & ecc & $\begin{array}{l}\text { inc } \\
(\mathrm{deg})\end{array}$ & $\begin{array}{c}H_{V} \\
(\mathrm{mag})\end{array}$ & $p_{V}$ & $\begin{array}{l}\text { Diameter } \\
(\mathrm{km})\end{array}$ \\
\hline O8083 & 3.324 & 0.614 & 23.341 & 16.1 & $0.126 \pm 0.072$ & $2.358 \pm 0.508$ \\
\hline O8590 & 2.911 & 0.698 & 52.363 & 16.6 & $0.020 \pm 0.008$ & $4.366 \pm 0.979$ \\
\hline f0778 & 2.910 & 0.720 & 54.500 & 18.1 & $0.048 \pm 0.032$ & $1.457 \pm 0.570$ \\
\hline f 3421 & 2.889 & 0.595 & 17.408 & 18.3 & $0.023 \pm 0.018$ & $1.900 \pm 0.776$ \\
\hline f6567 & 3.120 & 0.658 & 21.107 & 17.1 & $0.036 \pm 0.011$ & $2.538 \pm 0.235$ \\
\hline f6694 & 3.059 & 0.701 & 20.525 & 17.6 & $0.031 \pm 0.007$ & $2.292 \pm 0.281$ \\
\hline f8929 & 2.893 & 0.640 & 15.204 & 17.0 & $0.137 \pm 0.027$ & $1.432 \pm 0.021$ \\
\hline K00H74D & 2.919 & 0.598 & 49.262 & 18.0 & $0.163 \pm 0.029$ & $0.827 \pm 0.015$ \\
\hline K03U12L & 3.291 & 0.701 & 19.737 & 17.2 & $0.200 \pm 0.051$ & $1.078 \pm 0.016$ \\
\hline K09W06O & 3.086 & 0.581 & 28.760 & 17.4 & $0.034 \pm 0.008$ & $2.490 \pm 0.014$ \\
\hline K09WA4F & 3.073 & 0.659 & 17.007 & 17.3 & $0.047 \pm 0.009$ & $2.226 \pm 0.032$ \\
\hline $\mathrm{K} 10 \mathrm{~A} 79 \mathrm{G}^{\mathrm{a}}$ & 2.905 & 0.579 & 32.959 & 19.9 & $0.018 \pm 0.003$ & $0.892 \pm 0.009$ \\
\hline $\mathrm{K} 10 \mathrm{D} 21 \mathrm{M}^{\mathrm{a}}$ & 2.863 & 0.657 & 21.151 & 20.2 & $0.133 \pm 0.024$ & $0.303 \pm 0.012$ \\
\hline $\mathrm{K} 10 \mathrm{D} 77 \mathrm{H}^{\mathrm{a}}$ & 3.265 & 0.709 & 34.378 & 21.8 & $0.010 \pm 0.002$ & $0.574 \pm 0.017$ \\
\hline K10G62X & 2.953 & 0.705 & 21.658 & 20.1 & $0.014 \pm 0.002$ & $0.802 \pm 0.010$ \\
\hline K10P66R & 2.927 & 0.686 & 17.571 & 19.3 & $0.068 \pm 0.014$ & $0.678 \pm 0.021$ \\
\hline $\mathrm{K}_{10 \mathrm{U} 08 \mathrm{~B}^{\mathrm{a}}}$ & 2.984 & 0.627 & 30.968 & 19.7 & $0.032 \pm 0.026$ & $0.850 \pm 0.334$ \\
\hline $\mathrm{K} 11 \mathrm{~B} 59 \mathrm{~N}^{\mathrm{a}}$ & 3.063 & 0.622 & 20.323 & 20.4 & $0.013 \pm 0.009$ & $0.994 \pm 0.400$ \\
\hline K14A33A & 2.890 & 0.662 & 20.074 & 19.3 & $0.054 \pm 0.013$ & $0.787 \pm 0.036$ \\
\hline $\mathrm{K} 14 \mathrm{M} 18 \mathrm{Q}^{\mathrm{a}}$ & 2.896 & 0.600 & 35.087 & 15.6 & $0.037 \pm 0.066$ & $5.272 \pm 3.496$ \\
\hline $\mathrm{K} 14 \mathrm{O} 01 \mathrm{Z}^{\mathrm{a}}$ & 2.853 & 0.602 & 21.338 & 21.0 & $0.013 \pm 0.025$ & $0.732 \pm 0.292$ \\
\hline K14R12L & 2.771 & 0.671 & 23.605 & 17.9 & $0.256 \pm 0.039$ & $0.693 \pm 0.026$ \\
\hline $\mathrm{K} 14 \mathrm{X} 07 \mathrm{X}^{\mathrm{a}}$ & 2.901 & 0.597 & 36.714 & 19.7 & $0.015 \pm 0.017$ & $1.198 \pm 0.379$ \\
\hline
\end{tabular}

Note.

${ }^{\text {a }}$ Objects discovered by NEOWISE.
2. ecc $>0.55$
3. inc $>15^{\circ}$
4. inc $>90-\frac{90 a}{3.4}$
5. inc $>90-\frac{90 \mathrm{ecc}}{0.7}$
6. $1.8<T_{\mathrm{J}}<2.5+\frac{0.5 q}{1.2}$
7. $\left|z_{\text {peri }}\right|>0.15$ AU.

After identifying the region of orbital element-space that is most likely to be populated by escaped members of the Euphrosyne family, we apply these limits to search all currently known NEOs. Of the $\sim 12,000$ known NEOs to date, we find 113 asteroids that fulfill our selection criteria. Of these, 23 objects had diameters and albedos measured by the WISE or NEOWISE missions (Mainzer et al. 2011b, 2012b, 2014; Nugent et al. 2015), which we list in Table 1. As the WISE and NEOWISE surveys were conducted at thermal infrared wavelengths, they are effectively unbiased with respect to albedo, and should fairly sample both high and low albedo objects on a given orbit. In Figure 10 we compare the overall distribution of albedos for all NEOs to the distribution of albedos of our candidate objects, as well as that of all Main Belt asteroids associated with the Euphrosyne family (Masiero et al. 2013). Low albedos are over-represented among candidate family members when compared to the overall NEO population, however the albedos of these objects do not uniformly match that of the family. Given that the residence time of Euphrosyne family members in the NEO population is shorter than that of most NEOs, it is not surprising that the family does not dominate even this high-likelihood region. We note that albedos from the post-cryo and restarted NEOWISE missions have significant systematic uncertainties that may artificially

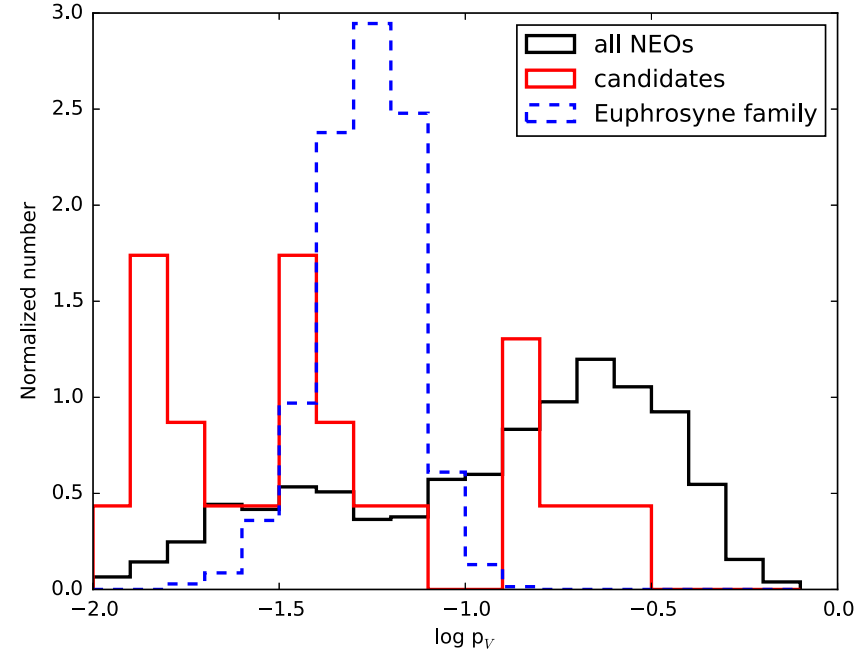

Figure 10. Distribution of the geometric optical albedos $\left(p_{V}\right)$ of all NEOs observed by WISE/NEOWISE (black solid, $N=662$ ), the subset of those NEOs identified as candidate escapees from the Euphrosyne family (red solid, $N=23$ ), and all Main Belt asteroids associated with the Euphrosyne family (blue dashed, $N=1392$ ).

broaden the distribution with respect to the albedos measured in the cryogenic portion of the NEOWISE survey.

Interestingly, the low albedo objects among the candidates are in general darker than the albedo distribution of members of the Euphrosyne family, which correspond to the low albedo peak seen for the outer Main Belt. Caution is needed in interpreting this result, as our sample does suffer from smallnumber statistics and detection biases, as well as potential contamination from other source regions. However, if these 
candidates are indeed escapees from the Euphrosyne family we would need to identify a plausible explanation for the difference in albedo from the family. A potential avenue is the investigation of space weathering effects on low albedo asteroids or changes in optical properties due to changing surface regolith size. Recent work comparing colors and albedos of C-type families has indicated that low albedo asteroids become redder and darker with time, but that smaller C-type asteroids may have lower albedos than larger ones (Kaluna et al. 2015). Investigation of these effects would benefit from further characterization of the physical properties of NEOs, particularly albedos and spectra.

We also used the limits derived from our simulations to search the NEO population model of Greenstreet et al. (2012). This model tabulates the fractional representation of a debiased NEO population across near-Earth space, tracing bins back to one of five NEO source regions. Using the above limits (except for the $z_{\text {peri }}$ limit as this information is not available in the Greenstreet model), we find that less than $4 \%$ of simulated NEOs are predicted to be found in our region of interest, and of those only $3 \%$ originated from the $\nu_{6}$ resonance. We note that the simulations of Greenstreet et al. (2012) follow Bottke et al. (2002) and only consider the region of the Main Belt near the $\nu_{6}$ resonance at semimajor axes $a<2.5 \mathrm{AU}$. The majority $(54 \%)$ of objects in this region are predicted to evolve into the NEO region from the Jupiter-family comets (JFCs), while $25 \%$ originated in the outer Main Belt. Again following Bottke et al. (2002), the outer Main Belt region tested by Greenstreet et al. (2012) was limited to inclinations $i<15^{\circ}$, significantly below our the initial conditions for our simulations. Bottke et al. (2002) do include this dynamical region in their MB2 population, however they state that it is only a minor contributor to the NEO population. We note that the statistically determined percentage of objects in this region originating from JFCs matches the estimates of the cometary contribution to the NEOs with $T_{\mathrm{J}}<3$ by DeMeo \& Binzel (2008) of $\sim 54 \% \pm 10 \%$ based on observed physical properties.

Given the simulations of Greenstreet et al. (2012), it is possible that our candidate list is significantly contaminated by inactive JFCs. Lamy et al. (2004) show that the albedos of bare JFC nuclei typically range from 0.02 to 0.04 , which is lower than the albedos of Euphrosyne family members $\left(p_{V}=0.06 \pm 0.02\right)$. Using a thermal infrared survey conducted with the Spitzer Space Telescope, Fernandez et al. (2013) found that the median diameter of JFCs is $\sim 2-5 \mathrm{~km}$, similar in size to the majority of the test particles in our simulations, though with a cumulative size frequency distribution (SFD) slope of $\alpha=-1.9 \pm 0.2$ where the SFD is described as $N_{>D} \propto D^{\alpha}$. Preliminary NEOWISE results on a larger sample of comet nuclei confirm this shallow SFD (Bauer et al. 2015). This value is significantly shallower than the present day Euphrosyne family, which has an SFD slope of $\alpha=-4.40 \pm 0.05$ (Masiero et al. 2013), our initialized family SFD from Carruba et al. (2014) of $\alpha=-3.6$, or the SFD of the near-Earth object population created in our simulations $(\alpha=-3.81 \pm 0.02)$. Thus JFCs may explain part of lowest albedo objects in our candidate albedo distribution, however given the shallower size distribution we would expect the relative contribution of JFCs to decrease for smaller objects.

We also compare our candidate source region to the objects expected from the debiased NEO population of Mainzer et al. (2012a). Given the semimajor axis limits listed above, the region of interest falls entirely within the Amor subpopulation (objects with perihelia $1.0<q<1.3 \mathrm{AU}$ ). Applying the cuts derived from our simulations to the debiased population shows that we should expect approximately $2.3 \pm 0.2 \%$ of all Amor NEOs and $0.8 \pm 0.2 \%$ of all NEOs to have orbital elements consistent with family member candidates. Low albedo NEOs $\left(p_{V}<0.1\right)$ make up $39.1 \pm 0.7 \%$ of all NEOs in the debiased population and $41.7 \pm 0.7 \%$ of the debiased Amor population. If our candidate objects all had low albedos they could account for the majority of the excess of dark asteroids in the Amor population when compared to the general NEO population. As seen in Figure 10, however, the candidate objects with measured albedos show some contamination from high albedo asteroids, thus we consider it unlikely that refugees from the Euphrosyne family explain the difference between the Amor and NEO albedo distributions completely.

La Spina et al. (2004) showed that NEOs have spin states that are preferentially retrograde, a hallmark of their evolution under Yarkovsky driving them inward toward the $\nu_{6}$ resonance where it intersects the inner Main Belt. As the Euphrosyne family has overlapped the $\nu_{6}$ resonance from formation, we would not expect a similar selection effect in the rotation poles by Yarkovsky mobilization, and instead would expect family members to show equal populations of prograde and retrograde rotators. None of the objects in our candidate list have published rotation poles in the Asteroid Lightcurve Database (Warner et al. 2009), however future surveys may provide insight into the rotation properties of this population.

\section{CONCLUSIONS}

The Euphrosyne family is one of the largest low albedo families known, and its unique location in the outer Main Belt overlapping the $\nu_{6}$ resonance means that its contribution to the NEO population has a unique signature. Our simulations of asteroid orbital evolution over the age of the family allow us to determine the best region of orbital element-space to search for candidate family members among the NEOs. The most probable region is found near the semimajor axis of the family, at large inclinations and very large eccentricities. This results in a Jupiter Tisserand parameter similar to what is observed for Jupiter-family comets. We also find that candidate family members typically have large vertical distances from the ecliptic at perihelion, as opposed to the majority of known NEOs that tend to reach perihelion close to the ecliptic plane (although we note this is almost undoubtedly biased by observational selection effects favoring detection of NEOs close to the Earth and thus near the ecliptic plane).

Using the limits derived here, we identify the currently known NEOs that are most likely to have evolved from the Euphrosyne breakup into near-Earth orbits, $\sim 1 \%$ of total population. Approximately $20 \%$ of candidates have diameter and albedo measurements in at least one phase of the NEOWISE mission, allowing direct comparison to the Euphrosyne family, and the overall NEO population. We find that candidates are preferentially lower in albedo than the overall NEO population. Many candidates also are darker than the Euphrosyne family, which may be indicative of the contribution of dead comets from the JFC population into the NEOs in this region of orbital element space. Followup observations of colors and spectral properties would help differentiate dormant/dead comets from Euphrosyne family 
members. As the Euphrosyne family has a steeper size distribution than JFCs, future surveys of NEOs to smaller sizes (especially thermal infrared surveys that are not biased against low albedo objects) would have a high likelihood of discovering a significant number of escapees from this family.

This research was carried out at the Jet Propulsion Laboratory, California Institute of Technology, under a contract with the National Aeronautics and Space Administration. J. M. was funded by a NASA Planetary Geology and Geophysics grant, and through the JPL internal Research and Technology Development program. V. C. was supported by the FAPESP grant 2014/06762-2. The authors would like to thank UNESP, CAPES, AAB, and FAPESP for supporting the 2014 Small Bodies Dynamics conference in Ubatuba, Brazil, which inspired this work. The JPL High-Performance Computing Facility used for our simulations is supported by the JPL Office of the CIO. This publication makes use of data products from the Wide-field Infrared Survey Explorer, which is a joint project of the University of California, Los Angeles, and the Jet Propulsion Laboratory/California Institute of Technology, funded by the National Aeronautics and Space Administration. This publication also makes use of data products from NEOWISE, which is a project of the Jet Propulsion Laboratory/California Institute of Technology, funded by the Planetary Science Division of the National Aeronautics and Space Administration.

\section{REFERENCES}

Bauer, J. M., Stevenson, R., Kramer, E., et al. 2015, ApJ, submitted Bottke, W. F., Morbidelli, A., Jedicke, R., Petit, J.-M., et al. 2002, Icar, 156,399
Bottke, W. F., Vokrouhlický, D., Rubincam, D. P., \& Nesvorný, D. 2006, AREPS, 34, 157

Bottke, W. F., Vokrouhlický, D., Walsh, K. J., et al. 2015, Icar, 247, 191

Brož, M. 2006, PhD thesis, Charles University, http://sirrah.troja.mff.cuni.cz/ $\sim$ mira $/ \mathrm{mp} / \mathrm{phdth} /$

Bus, S. J., \& Binzel, R. P. 2002, Icar, 158, 146

Carruba, V., Aljbaae, S., \& Souami, D. 2014, ApJ, 792, 46

Carruba, V., Masiero, J., Cibulkova, H., Aljbaae, S., \& Huaman, M. 2015, in IAU General Assembly, Meeting \#29 (Washington, D.C.: AAS), 2247929

Carry, B. 2012, P\&SS, 73, 98

DeMeo, F. E., \& Binzel, R. P. 2008, Icar, 194, 436

Fernandez, Y., Kelley, M. S., Lamy, P. L., et al. 2013, Icar, 226, 1138

Gladman, B., Michel, P., \& Froeschle, C. 2000, Icar, 146, 176

Greenstreet, S., Ngo, H., \& Gladman, B. 2012, Icar, 217, 355

Holman, M. J., \& Wisdom, J. 1993, AJ, 105, 1987

Kaluna, H. M., Meech, K. J., \& Masiero, J. R. 2015, Icar, submitted

Kozai, Y. 1962, AJ, 67, 591

La Spina, A., Paolicchi, P., Kryszczynska, A., \& Pravec, P. 2004, Natur, 428, 400

Lamy, P. L., Toth, I., Fernandez, Y. R., \& Weaver, H. A. 2004, in Comets II, ed. M. C. Festou, H. U. Keller \& H. A. Weaver (Tucson: Univ. Arizona Press), 223

Levison, H. F. 1996, in ASP Conf. Proc. 107, Completing the Inventory of the Solar System (San Francisco, CA: ASP), 173

Levison, H. F., \& Duncan, M. J. 1994, Icar, 108, 18

Mainzer, A., Grav, T., Bauer, J. M., et al. 2011a, ApJ, 743, 156

Mainzer, A. K., Bauer, J. M., Cutri, R. M., et al. 2014, ApJ, 792, 30

Mainzer, A. K., Bauer, J. M., Grav, T., et al. 2011b, ApJ, 731, 53

Mainzer, A. K., Grav, T., Masiero, J., et al. 2012a, ApJ, 752, 110

Mainzer, A. K., Grav, T., Masiero, J., et al. 2012b, ApJL, 760, 12

Masiero, J. R., Grav, T., Mainzer, Nugent, C., et al. 2014, ApJ, 791, 121

Masiero, J. R., Mainzer, A. K., Bauer, J. M., et al. 2013, ApJ, 770, 7

Milani, A., Cellino, A., Knežević, Z., et al. 2014, Icar, 239, 46

Nesvorný, D. 2012, Nesvorny HCM Asteroid Families V 2.0. NASA Planetary Data System, EAR- A-VARGBDET-5-NESVORNYFAM-V2.0.

Nugent, C. R., Mainzer, A., Masiero, J., et al. 2015, APJ, Submitted

Vokrouhlický, D., Brož, M., Bottke, W. F., Nesvorný, D., \& Morbidelli, A. 2006, Icar, 182, 118

Warner, B. D., Harris, A. W., \& Pravec, P. 2009, Icar, 202, 134 